\title{
Affective auditory stimuli: Characterization of the International Affective Digitized Sounds (IADS) by discrete emotional categories
}

\author{
Ryan A. STEVenson and Thomas W. James \\ Indiana University, Bloomington, Indiana
}

\begin{abstract}
Although there are many well-characterized affective visual stimuli sets available to researchers, there are few auditory sets available. Those auditory sets that are available have been characterized primarily according to one of two major theories of affect: dimensional or categorical. Current trends have attempted to utilize both theories to more fully understand emotional processing. As such, stimuli that have been thoroughly characterized according to both of these approaches are exceptionally useful. In an effort to provide researchers with such a stimuli set, we collected descriptive data on the International Affective Digitized Sounds (IADS), identifying which discrete categorical emotions are elicited by each sound. The IADS is a database of 111 sounds characterized along the affective dimensions of valence, arousal, and dominance. Our data complement these characterizations of the IADS, allowing researchers to control for or manipulate stimulus properties in accordance with both theories of affect, providing an avenue for further integration of these perspectives. Related materials may be downloaded from the Psychonomic Society Web archive at www.psychonomic.org/archive.
\end{abstract}

Historically, studies of emotion have been carried out primarily in the visual realm. In recent years though, there have been a growing number of experiments using audio stimuli as a means to study emotion, both as unisensory stimuli and as part of multisensory stimuli. Because this is a more recent trend than using a vision-only approach, there is a significant gap between the availability of wellcharacterized audio and visual stimuli in the scientific cotmmunity. Many visual stimulus sets including the International Affective Picture Set (IAPS; Lang, Bradley, \& Cuthbert, 2005), the Affective Norms for English Words (ANEW; Bradley \& Lang, 1999a), and the Pictures of Facial Affect (POFA; Ekman \& Friesen, 1975) have all been characterized according to both of the two predominant theories used to describe emotion, the dimensional and discrete category approaches (Mikels et al., 2005; Stevenson \& James, 2007; Stevenson, Mikels, \& James, 2007). This is not the case with auditory stimuli.

A few auditory stimuli sets have been standardized according to the dimensional theories of emotion independent of emotional category. One of these is the International Affective Digitized Sounds (IADS), a set of 111 standardized, emotionally evocative sounds that cover a wide range of semantic categories. This system was created with three goals in mind: better experimental control of emotional stimuli, increasing the ability of cross-study comparisons of results, and increased ability to directly replicate studies (Bradley \& Lang, 1999b). To achieve these goals, the IADS were originally normalized using the Self-Assessment Manikin (SAM), a scale that assesses valence, arousal, and dominance as dimensions describing emotion (Bradley \& Lang, 1994).

The dimensional theories of emotion propose that affective meaning can be well characterized by a small number of dimensions. Dimensions are chosen on their ability to statistically characterize subjective emotional ratings with the least number of dimensions possible (Bradley \& Lang, 1994). These dimensions generally include one bipolar or two unipolar dimensions that represent positivity and negativity, and have been labeled in various ways, such as valence or pleasure. Also usually included is a dimension that captures intensity, arousal, or energy level.

The IADS, as well as numerous other sound collections, have been used successfully with characterizations of valence and arousal, two dimensions that have been shown to make dimensional theories of affect most powerful (Mehrabian \& Russell, 1974; Smith \& Ellsworth, 1985; Yik, Russell, \& Barrett, 1999), as well as dominance. Neural activity has been identified as responding preferentially with positive or negative sounds as opposed to neutral sounds (Frey, Kostopoulos, \& Petrides, 2000; Royet et al., 2000) as well as evoking different psychophysiological responses, including heart rate, skin conductance, and respiration rate (Gomez \& Danuser, 2004). Differences in these responses can be seen in psychopathic individuals who have abnormal reactions to both positive and negative emotional sounds 
(see, e.g., Verona, Patrick, Curtin, Bradley, \& Lang, 2004). Differences in response to affective auditory stimuli can also be seen with other conditions as well, even with irritable bowel syndrome (Andresen et al., 2006)!

In contrast to the dimensional theories, categorical theories claim that the dimensional models, particularly those using only two or three dimensions, do not accurately reflect the neural systems underlying emotional responses. Instead, supporters of these theories propose that there are a number of emotions that are universal across cultures and have an evolutionary and biological basis (Ekman, 1992). Which discrete emotions are included in these theories is a point of contention, as is the choice of which dimensions to include in the dimensional models. Most supporters of discrete emotion theories agree that at least the five emotions of happiness, sadness, anger, fear, and disgust should be included.

Studies of emotion using acoustic stimuli have also had empirical success using these discrete categorical theories of affect. Changes in facial electromyography (EMG) have been reported with different emotions (Jäncke, Vogt, Musial, Lutz, \& Kalveram, 1996), as well as behavioral changes, such as decoding accuracy (Juslin \& Laukka, 2001). In addition to these peripheral methods of measuring responses to categorical stimuli, clear differences can be seen in brain activation patterns with stimuli that are equivalent according to dimension, yet fall into different emotional categories. Distinct neural patterns have been shown for categorical emotions using electroencephalography (EEG; Hans, Eckart, \& Hermann, 1997), positron emission topography (PET; George et al., 1996; Imaizumi et al., 1997), and functional magnetic resonance imaging (fMRI; Buchanan et al., 2000; Dolan, Morris, \& de Gelder, 2001; Ethofer et al., 2006; Grandjean et al., 2005; Imaizumi et al., 1997; Sander, Brechmann, \& Scheich, 2003; Sander \& Scheich, 2001). Brain damage or lesions have also been shown to affect the perception of specific emotional categories. The most compelling of these is a study by Scott et al. (1997) that describes a brain damaged patient who shows impaired responses on emotional recognition tasks using sounds and prosody, but only when the stimuli were angry or fearful, not when they were happy, sad, or disgusting. Many other studies have shown brain damage or lesion patients to have altered perception of emotional sounds, such as prosody (Adolphs, Damasio, \& Tranel, 2002; Adolphs, Tranel, \& Damasio, 2001; Baum \& Dwivedi, 2003; Gandour, Larsen, Dechongkit, Ponglorpisit, \& Khunadorn, 1995; Kujala, Lepistö, Nieminen-von Wendt, Näätänen, \& Näätänen, 2005) and music (Gosselin, Peretz, Johnsen, \& Adolphs, 2007). Likewise, it has been shown that virtual lesions created by repetitive transcranial magnetic stimulation (TMS) can selectively impair recognition of withdrawal emotions, such as fear and sadness, while leaving responses to approach emotions, such as happiness and anger, unaltered (van Rijn et al., 2005).

The data from these studies provide strong evidence for different biological systems underlying these emotional categories that cannot be accounted for by dimensional models. The purpose of dimensional models is to mathematically account for the most variability in subjective rat- ings using the smallest number of dimensions (Bradley \& Lang, 1994). Although it may be the case that, mathematically, two or three dimensions can more parsimoniously account for the variability in subjective ratings, it does not imply that these dimensions necessarily characterize the underlying biological processes involved. Both are models with multiple factors (dimensions or categories) that, when weights are applied, can characterize specific emotions. The choice of factors is based on theoretical preference. Here is the main difference between dimensional and categorical models: In the case of dimensional models, the choice of factors is based on mathematical parsimony (Bradley \& Lang, 1994), whereas, for so-called discrete categorical models, the choice of factors is based on biological or evolutionary processes (Ekman, 1992). In fact, the different theoretical basis for the models can be seen in the results of previous studies. Experiments based on dimensional models do a good job of accounting for variability in subjective ratings and some peripheral psychophysiological measures, as described above. On the other hand, experiments based on categorical models have been much more successful in accounting for brain activation patterns, as previously described. Although the data provided by this study will be of value to all researchers studying sounds stimuli with emotional valence, it may be of most use to researchers studying emotional sounds with respect to patterns of brain activation.

Dimensional and categorical theories of affect, although both effective characterizations of emotion, are not mutually exclusive. Many researchers who subscribe to the dimensional model view the positive and negative valence systems as appetitive and defensive systems, respectively (Bradley, Codispoti, Cuthbert, \& Lang, 2001; Bradley, Codispoti, Sabatinelli, \& Lang, 2001), with arousal representing the intensity of activation within each system. Commonly used visual stimuli, such as the IAPS (Lang et al., 2005), which were originally described in accord with the dimensional approach, produce different responses in skin conductance, startle reflex, and heart rate depending on category (Bradley, Codispoti, Cuthbert, \& Lang, 2001; Bradley, Codispoti, Sabatinelli, \& Lang, 2001). Also, categorical approaches have begun to incorporate intensity or arousal into their models, showing changes in responses with higher intensity sounds within given emotional categories behaviorally (Juslin \& Laukka, 2001), in facial EMG (Jäncke et al., 1996), and in associated neural responses seen with fMRI (Ethofer et al., 2006). With these empirical overlaps in theories of affect, visual stimuli previously only characterized according to a single affective theory have now been characterized according to the complimentary theory, including the IAPS (Mikels et al., 2005), the ANEW (Stevenson et al., 2007), and the POFA (Stevenson \& James, 2007), with stimuli from each of these sets eliciting category specific responses. Characterizations according to both theories of affect can be useful, providing researchers with a more complete characterization of affect.

In an effort to provide a set of well-characterized affective stimuli in the auditory sensory modality, we have collected discrete emotional category ratings for the IADS, 
which has previously been characterized according to the dimensional theory of affect (Bradley \& Lang, 1999b). Due to the empirical success of auditory stimuli in studies relying on either categorical or dimensional theories of affect, it follows that stimuli characterized by both have the potential to be even more informative. These data, in conjunction with the original characterization along the dimensions of valence, arousal, and dominance, provide a set of auditory stimuli with a more complete description of affective properties of each sound, and will open up new possibilities to researchers. With these new norms, we provide a tool with which experimenters can control both dimensional and categorical aspects of their emotional sound stimuli. This control will allow researchers to create more homogenous stimulus sets that can be more precisely and rigorously defined with regard to not only valence, arousal, and dominance, but also with regard to discrete emotional category characteristics. In addition, experiments in which stimuli have been characterized according to both dimensional and discrete categorical theories of emotion will provide a unique opportunity to analyze experiments according to both theories, providing a means to further investigate which theory, or which aspects of each theory, better describe the components of emotional responses.

\section{METHOD}

\section{Participants}

Eighty participants ( 40 female, mean age $=19.6$ ) received course credit for participation. The experiment protocol was approved by the Indiana University Committee for the Use of Human Subjects in Research.

\section{Design and Procedure}

Participants were presented each of the 111 sounds in the IADS using MATLAB 5.2 (MathWorks, Inc., Natick, MA) software with the Psychophysics Toolbox extensions (Brainard, 1997; Pelli, 1997) running on a Macintosh computer, through Beyerdynamic DT 100 headphones. Each stimulus's maximum RMS was adjusted to 1 and presented at full volume. No participants reported having any difficulty hearing the sounds. Stimuli were presented in a random order for each participant. Following the sound, participants saw a series of five rating scales including happiness, anger, sadness, fear, and disgust, with scales presented in random orders. These emotions were chosen for two reasons: their inclusion in nearly all discrete categorical theories of emotion, and their inclusion in databases of facial expression. (Although surprise is included in many facialexpression stimuli sets, it is not as commonly included in theories of discrete emotion and, as such, was not included.) Participants rated each sound on all five emotions independently, with each discrete emotional scale ranging from 1 to $9-1$ being not at all and 9 being extremely. Participants had $1 \mathrm{~h}$ to complete all ratings. In the case that participants did not finish within $1 \mathrm{~h}$, only sounds for which all five ratings had been given were scored, resulting in 71-75 scores for each sound $(M=73.7)$.

Means and standard deviations of the five ratings were calculated individually for each sound (see archived supplementary materials). According to these means and standard deviations, category labels were created for each sound at two different levels. At the first level, single emotion categories were defined where one emotion was one or more standard deviations higher than all four other emotions. A dual emotion label (e.g., anger and fear) was given to sounds whose response to two emotions were one or more standard deviations above the remaining three, but not more than one above each other, and so on. Sounds in which all four negative emotions were one or more standard deviations above the happiness rating, but not above each other, were labeled negative. If none of these criteria were met, no label was given. The second level reported was done using the same categories, but was based on a more liberal criterion of 0.5 standard deviations (see archived supplementary materials).

\section{RESULTS}

In order to ascertain whether or not these categorical data could be extrapolated from the previously collected dimensional data, regressions were run using the discrete emotional category ratings collected from all participants and sounds to predict the previous ratings for valence, arousal, and dominance (Bradley \& Lang, 1999b). Prior to regression analysis, sounds were separated into negative (valence rating $<5$ ) and positive (valence rating $>5$ ) groups in order to obtain results that were comparable to previous studies. Six regressions were run using the five emotional category ratings to predict valence, arousal, and dominance within both the positive and negative groups of words. Standardized $\beta$ coefficients were calculated for all five emotional categories (Table 1), with Bonferronicorrected for family-wise error $p$ values less than .0017

Table 1

Regressions of Discrete Emotional Category Ratings Predicting Valence, Arousal, and Dominance for Negative and Positive Sounds

\begin{tabular}{|c|c|c|c|c|c|c|c|}
\hline & & \multicolumn{2}{|c|}{ Predicting Valence } & \multicolumn{2}{|c|}{ Predicting Arousal } & \multicolumn{2}{|c|}{ Predicting Dominance } \\
\hline & & $\beta$ & $t$ & $\beta$ & $t$ & $\beta$ & $t$ \\
\hline Negative valence sounds & $\begin{array}{l}\text { happiness } \\
\text { fear } \\
\text { anger } \\
\text { disgust } \\
\text { sadness }\end{array}$ & $\begin{array}{r}.361 \\
-.415 \\
-.069 \\
-.056 \\
-.046\end{array}$ & $\begin{aligned} & 3.268^{\circ \circ} \\
&-2.779^{\circ}-0.485 \\
&-0.523 \\
&-0.346\end{aligned}$ & $\begin{array}{r}-.460 \\
.847 \\
.068 \\
-.126 \\
-.164\end{array}$ & $\begin{array}{c}-0.424 \\
5.729 \\
0.483 \\
-1.190 \\
-1.241\end{array}$ & $\begin{array}{r}.279 \\
-.873 \\
.092 \\
.085 \\
.224\end{array}$ & $\begin{array}{c}2.787^{*} \\
-6.437^{* * *} \\
0.713 \\
0.872 \\
1.853\end{array}$ \\
\hline Positive valence sounds & $\begin{array}{l}\text { happiness } \\
\text { fear } \\
\text { anger } \\
\text { disgust } \\
\text { sadness }\end{array}$ & $\begin{array}{r}.815 \\
-.073 \\
.016 \\
.017 \\
.065\end{array}$ & $\begin{array}{r}9.167^{* * *} \\
-0.741 \\
0.162 \\
0.215 \\
0.794\end{array}$ & $\begin{array}{r}.740 \\
.398 \\
.214 \\
.279 \\
-.150\end{array}$ & $\begin{array}{c}6.989^{* * *} \\
3.373^{* * *} \\
1.847 \\
2.910^{* *} \\
-1.532\end{array}$ & $\begin{array}{r}.257 \\
-.768 \\
.236 \\
.074 \\
-.033\end{array}$ & $\begin{array}{c}2.733^{*} \\
-7.338^{* * *} \\
2.294^{*} \\
0.865 \\
-0.384\end{array}$ \\
\hline
\end{tabular}

Note- $\beta$ values, $t$ scores, and significance levels are shown for each emotional category with respect to each emotional dimension. After Bonferroni corrections for family-wise error, corrected $p$ values less than .0017 should be interpreted as being statistically significant. " $p<.05$. " $p<.005$. ${ }^{* * *} p<.001$. 
deemed significant. With negative words, valence was marginally predicted by happiness $(p<.002)$ and fear $(p<.008)$; arousal was significantly predicted by fear $(p<.001)$; dominance was significantly predicted by fear $(p<.001)$ and marginally predicted by happiness $(p<$ .008 ). With positive words, valence was significantly predicted by happiness $(p<.001)$; arousal was significantly predicted by happiness $(p<.001)$ and fear $(p<.001)$, and marginally predicted by disgust $(p<.005)$; dominance was significantly predicted by fear $(p<.001)$, and marginally predicted by happiness $(p<.009)$ and anger $(p<.026)$.

Regressions using the dimensional ratings to predict emotional category ratings were also run for the same reason. The regressions using the dimensional ratings to predict emotional category ratings were similar to the previous regressions, with a lack of homogeneity in the ability of categorical ratings to predict dimensional ratings. Standardized $\beta$ coefficients were calculated for all three emotional dimensions (Table 2), with Bonferroni-corrected for family-wise error $p$ values less than .0017 deemed significant. With negative sounds, happiness was significantly predicted by valence $(p<.001)$ and marginally predicted by arousal $(p<.005)$; fear was not significantly or marginally predicted by any dimension; neither anger, disgust, nor sadness were significantly predicted by any dimension, but all were marginally predicted by valence $(p<.05, p<.005, p<.005$, respectively). With positive sounds, happiness was significantly predicted by valence $(p<.001)$; fear was significantly predicted by dominance $(p<.001)$ and marginally predicted by arousal $(p<.05)$; neither anger, disgust, nor sadness were significantly predicted by any dimension, though anger was marginally predicted by valence $(p<.05)$ and arousal $(p<.05)$, disgust by valence $(p<.005)$, arousal $(p<.05)$, and dominance $(p<.05)$, and sadness by arousal $(p<.05)$ and dominance $(p<.05)$.

Responses were analyzed according to sex for each emotional category and individual sound. Means and standard deviations were calculated for female and male ratings independently (see archived supplementary materials). $t$ tests were run on ratings for each individual word for all five discrete emotional categories. No sounds showed any significant differences between male and female ratings.

Categorical labels did differ for males and females with eight sounds (7.2\%) at the one standard deviation level, and five sounds $(4.5 \%)$ at the 0.5 standard deviation level of categorization. In these cases, ratings on a given category met the requirements of labeling for one sex and not for the other (i.e., a sound labeled sad for females and not labeled for males). It was never the case that the sounds were categorically labeled as different emotions for males and females (i.e., a sound labeled sad for females and disgusting for males). It should also be noted that these are not direct comparisons between the ratings from each sex, and as such, it would be inaccurate to interpret this difference in labeling as a sex difference in the mean ratings for any of these words.

\section{DISCUSSION}

This study provides categorical data that will allow the IADS to be used in studies of emotional categories, and will also provide a means of investigating the association of the dimensional and categorical approaches to the study of affect. The heterogeneity of effects that each emotional category has on different dimensional attributes of the stimuli is similar to that seen with the ANEW (Stevenson et al., 2007) and the POFA (Stevenson \& James, 2007). The consistency of these findings highlights the importance of using categorical data both independently and as a supplement to dimensional data.

\section{Emotional Categories}

Forty-two of the 111 sounds (37.8\%) met the abovementioned criterion to be labeled with a discrete emotional category at the one standard deviation level. This level, however, is arbitrary, and should not be considered the correct level at which to categorize these sounds. Given unique needs of individual investigators and experiments, a more conservative or more liberal criterion may be implemented using these data, including the ability to use sounds associated with single, blended, or undifferentiated emotions (e.g., see Mikels et al., 2005). In particular,

Table 2

Regressions of Emotional Dimensions Predicting Discrete Emotional Category Ratings of Happiness, Fear, Anger, Disgust, and Sadness for Negative and Positive Sounds

\begin{tabular}{|c|c|c|c|c|c|c|c|}
\hline & & \multicolumn{2}{|c|}{ Predicting Valence } & \multicolumn{2}{|c|}{ Predicting Arousal } & \multicolumn{2}{|c|}{ Predicting Dominance } \\
\hline & & $\boldsymbol{\beta}$ & $t$ & $\beta$ & $t$ & $\beta$ & $t$ \\
\hline Negative valence sounds & $\begin{array}{l}\text { happiness } \\
\text { fear } \\
\text { anger } \\
\text { disgust } \\
\text { sadness }\end{array}$ & $\begin{array}{r}.937 \\
.036 \\
-.655 \\
-.008 \\
-.860\end{array}$ & $\begin{array}{c}4.212^{* * *} \\
0.177 \\
-2.522^{*} \\
-3.544^{* *} \\
-3.251^{* *}\end{array}$ & $\begin{array}{r}.876 \\
.396 \\
.286 \\
-.329 \\
.019\end{array}$ & $\begin{array}{r}3.602^{* *} \\
1.791 \\
1.007 \\
-1.056 \\
0.066\end{array}$ & $\begin{array}{r}.510 \\
-.439 \\
.362 \\
.431 \\
.369\end{array}$ & $\begin{array}{r}1.932 \\
-1.830 \\
1.175 \\
1.276 \\
1.174\end{array}$ \\
\hline Positive valence sounds & $\begin{array}{l}\text { happiness } \\
\text { fear } \\
\text { anger } \\
\text { disgust } \\
\text { sadness }\end{array}$ & $\begin{array}{r}.816 \\
.025 \\
-.516 \\
-.575 \\
.269\end{array}$ & $\begin{array}{c}6.917^{\circ * *} \\
0.192 \\
-2.772^{*} \\
-3.006^{\circ} \\
1.363\end{array}$ & $\begin{array}{r}.109 \\
.242 \\
.381 \\
.412 \\
-.319\end{array}$ & $\begin{array}{r}1.206 \\
2.440^{*} \\
2.669^{*} \\
2.804^{*} \\
-2.106^{*}\end{array}$ & $\begin{array}{r}-.760 \\
-.798 \\
-.210 \\
.472 \\
-.388\end{array}$ & $\begin{array}{c}-0.737 \\
-7.060^{* * *} \\
-0.129 \\
2.820^{*} \\
-2.251^{*}\end{array}$ \\
\hline
\end{tabular}

Note $-\beta$ values, $t$ scores, and significance levels are shown for each emotional dimension with respect to each emotional category. After Bonferroni corrections for family-wise error, corrected $p$ values less than .0017 should be interpreted as being statistically significant. $" p<.05$. $* p<.005 . * * 0<.001$. 
these categories can be used in tandem with the dimensional ratings provided by Bradley and Lang (1999b) to better control the emotional aspects of a given stimulus.

As an example, consider an experimenter who would like a strong negative sound without bias toward any particular discrete emotion. Using only the dimensional ratings, the researcher could pick sounds 276 or 277 , both females screaming, and with very low valence ratings of 1.91 and 1.74, respectively, but he or she would have no a priori means to discern whether the response to these stimuli represents negative affect in general or a narrower response associated with only fear. Using the categorical data, the researcher would have the a priori knowledge that these two sounds are particularly fearful, and may evoke a different response than one that is, for example, an equally negative but sad sound. A better choice would be sound 278 or 279 . Both have valence, arousal, and dominance ratings similar to the two previously discussed sounds, but without one prevailing emotional category that may bias or confound the experimenter's results. Thus, by using these data as well as the dimensional characterizations of the sounds, the researcher could design a stronger study by utilizing negative sounds that are not biased toward one particular emotion or another, or when appropriate, by utilizing sounds that evoke one and only one discrete emotion.

\section{Emotional Categories and Dimensions}

In previous characterizations of affective stimuli including written words (Stevenson et al., 2007) and emotional faces (Stevenson \& James, 2007), it has been shown that emotional dimensions lack the ability to consistently predict discrete emotional category ratings, and likewise discrete category information is insufficient to consistently predict dimensional ratings. This is displayed in the results of regressions using both discrete and dimensional data to predict each other. As an example of this inconsistency in predicting dimensional values with categorical, or vice versa, we will look at the regression predicting valance. For negative sounds, both fear and happiness ratings were marginally predictive of valence, whereas anger, sadness, and disgust had a negative effect of valence: Generally, the higher the rating for each of these three categories, the more negative a sound is rated. This reverses with positive sounds though: The higher a sound was rated with anger, sadness, and disgust, the more positively that sound was rated. In addition, happiness was only significantly predictive for positive sounds. Although this is only one example, it is indicative of all of the regressions.

To further illuminate this aspect of the data, one can compare the results of these regressions with those produced by a similar characterization of the ANEW by Stevenson et al. (2007). Identical regressions were run comparing dimensional and categorical ratings, and quite different results emerged. Again, we will look at the regression predicting valance. With negative words in the ANEW, happiness proved to be a strong positive predictor of valence, and sadness and disgust were strong negative predictors of valence. For the IADS, however, valence was not predicted by sadness or disgust, but was predicted by fear. In fact, sadness was the strongest predictor of valence for negative words in the ANEW, even though it was not significantly predictive for valence at all in the negative sounds of the IADS.

There was only one exception to this lack of predictive ability. In each stimuli set that we have characterized, the IADS, the ANEW, and the POFA, fear has always been a good predictor of arousal, both for positive and negative stimuli.

These data also illustrate the inability of either the dimensional models or categorical models to fully describe the emotional characteristics of stimuli. This is particularly seen in the regression where dimensions are used to predict categories. The categories of anger, disgust, and sadness are not significantly predicted by any of the three dimensions, and likewise, anger, disgust, and sadness are not significantly predictive of valence, arousal, or dominance. This would suggest that the dimensions of valence, arousal, and dominance may be able to describe the emotions of fear and happiness, but are lacking in their ability to characterize other negative emotions.

The inability of emotional dimensions to consistently predict categorical data, and vice versa, suggests that the information described by emotional dimensions and emotional categories is not congruent, repetitive information. As such, it is important for researchers to take information from both characterizations into account when attempting to choose stimuli, especially in the case where only a single measure of emotion is supposed to be the manipulated independent variable.

\section{Sex Differences}

Sex differences across categorical ratings in previous studies have been small, from $4 \%-13 \%$ (Bradley, Codispoti, Sabatinelli, \& Lang, 2001; Mikels et al., 2005; Stevenson \& James, 2007; Stevenson et al., 2007). Nevertheless, a complete lack of sex differences for any of the individual sounds was quite surprising, as numerous psychophysiological studies have commonly shown sex differences to emotional stimuli using various methods, including $f M R I$ (e.g., Wrase et al., 2003), EEG (e.g., Schirmer, Kotz, \& Friederici, 2005), steady-state probe topography (e.g., Kemp, Silberstein, Armstrong, \& Nathan, 2004), and more peripheral measures such as heart rate, facial EMG, skin conductance, and startle effect (e.g., Bradley, Codispoti, Sabatinelli, \& Lang, 2001). However, because this current data is only of a descriptive nature, any attempt to theorize why there is a lack of sex differences in the IADS where there were slight differences in the IAPS and the ANEW would be complete speculation. Future studies are needed to investigate this matter further.

Despite the lack of sex difference with each individual rating, there were eight sounds that were labeled differently for males and females at the level of one standard deviation. Of the eight sounds whose label differed, all eight of them were not labeled according to the males' ratings, but were labeled according to the females' ratings. Furthermore, seven of the eight additional labels with the females' ratings included the fear category, with five fear-only labels, and two anger-fear labels. The one 
exception was a female erotic sound clip, to which males did not produce a label, and females' ratings classified as happy-disgusting (every other erotic female sound was rated happy-disgusting by both males and females). This pattern was similar at the more liberal 0.5 standard deviation level, with three of five differences involving fearful stimuli (two fear-only labels and one anger-fear label). Again, due to the descriptive nature of these data, a cause of this pattern of more fear-labeled sounds with females cannot be ascertained, but further studies are needed to provide more information on the topic.

\section{Future Uses}

As researchers continue to explore emotional processing and integrate the dimensional and categorical theories of affect, stimuli will be needed that can be controlled and manipulated according to both of these theories. This characterization of the IADS provides such a stimulus set. Also, it provides the first such stimulus set in the auditory realm. Given the more recent trend of pursuing this field through audio and multisensory means, this set will allow researchers to be more selective and precise in their stimuli selection, providing a means to conduct more controlled and refined experiments with more homogeneous stimuli. In addition, stimuli sets that have been well characterized and controlled according to both dimensional and discrete categorical theories of emotion allow researchers to directly assess which theory, or which components of each theory, provide more accurate accounts of human emotional responses.

\section{AUTHOR NOTE}

This research was supported in part by the Indiana METACyt Initiative of Indiana University, funded in part through a major grant from the Lilly Endowment, Inc. Thanks to Laurel Stevenson and Karin James, as well as Jennifer Willingham and Peter Cole, for their support and insights on this work and article. Correspondence concerning this article should be addressed to R. A. Stevenson, Department of Psychological and Brain Sciences, Indiana University, 1101 East Tenth St., Room 239, Bloomington, IN 47405 (e-mail: stevenra@indiana.edu).

\section{REFERENCES}

Adolphs, R., Damasio, H., \& Tranel, D. (2002). Neural systems for recognition of emotional prosody: A 3-D lesion study. Emotion, 2, 23-51.

AdolPhs, R., Tranel, D., \& Damasio, H. (2001). Emotion recognition from faces and prosody following temporal lobectomy. Neuropsychology, 15, 396-404.

Andresen, V., Poellinger, A., Tsrouya, C., Bach, D., Stroh, A., FOERChLER, A., ET AL. (2006). Cerebral processing of auditory stimuli in patients with irritable bowel syndrome. World Journal of Gastroenterology, 12, 1723-1729.

BAUM, S. R., \& DWIVEDI, V. D. (2003). Sensitivity to prosodic structure in left- and right-hemispheric-damaged individuals. Brain \& Language, 87, 278-289.

Bradley, M. M., Codispoti, M., Cuthbert, B. N., \& Lang, P. J. (2001). Emotion and motivation: I. Defensive and appetitive reactions in picture processing. Emotion, 1, 276-298.

Bradley, M. M., Codispoti, M., Sabatinelli, D., \& Lang, P. J. (2001). Emotion and motivation: II. Sex differences in picture processing. Emotion, 1, 300-319.

Bradley, M. M., \& LANG, P. J. (1994). Measuring emotion: The SelfAssessment Manikin and the semantic differential. Journal of Behavior Therapy \& Experimental Psychiatry, 25, 49-59.
Bradley, M. M., \& LaNG, P. J. (1999a). Affective norms for English words (ANEW): Stimuli, instruction manual and affective ratings. (Tech. Rep. No. C-1). Gainesville, FL: University of Florida.

Bradley, M. M., \& LANG, P. J. (1999b). International Affective Digitized Sounds (IADS): Stimuli, instruction manual and affective ratings (Tech. Rep. No. B-2). Gainesville, FL: University of Florida.

Brainard, D. H. (1997). The Psychophysics Toolbox. Spatial Vision, 10, 433-436.

Buchanan, T. W., Lutz, K., Mirzazade, S., Specht, K., Shah, N. J., ZILLES, K., \& JÄNCKE, L. (2000). Recognition of emotional prosody and verbal components of spoken language: An fMRI study. Cognitive \& Brain Research, 9, 227-238.

DOLAN, R. J., MORRIS, J. S., \& DE GELDER, B. (2001). Crossmodal binding of fear in voice and face. Proceedings of the National Academy of Sciences, 98, 10006-10010.

EKmAN, P. (1992). Are there basic emotions? Psychological Review, 99, 550-553.

EkMAN P., \& Friesen, W. V. (1975). Pictures of facial affect. Palo Alto, CA: Consulting Psychologist Press.

Ethofer, T., ANDERS, S., Wiethoff, S., Erb, M., Herbert, C., SAur, R., ET AL. (2006). Effects of prosodic emotional intensity on activation of associative auditory cortex. NeuroReport, 17, 249-253.

Frey, S., Kostopoulos, P., \& PeTrides, M. (2000). Orbitofrontal involvement in the processing of unpleasant auditory information. Eumpean Journal of Neunoscience, 12, 3709-3712.

Gandour, J., Larsen, J., Dechongkit, S., Ponglorpisit, S., \& Khunadorn, F. (1995). Speech prosody in affective contexts in Thai patients with right hemisphere lesions. Brain \& Language, 51, 422-443.

George, M. S., Parekh, P. I., Rosinsky, N., Ketter, T. A., KImbrell, T. A., Heilman, K. M., ET AL. (1996). Understanding emotional prosody activates right hemisphere regions. Archives of Neurology, $53,665-670$.

Gomez, P., \& Danuser, B. (2004). Affective and physiological responses to environmental noise and music. International Journal of Psychophysiology, 53, 91-103.

Gosselin, N., Peretz, I., Johnsen, E., \& Adolphs, R. (2007). Amygdala damage impairs emotion recognition from music. Neumpsychologia, 45, 236-244.

Grandiean, D., Sander, D., Pourtois, G., Schwartz, S., Seghier, M. L., Scherer, K. R., \& Vuilleumier, P. (2005). The voices of wrath: Brain responses to angry prosody in meaningless speech. $\mathrm{Na}$ ture Neumscience, 8, 145-146.

Hans, P., Eckart, A., \& Hermann, A. (1997). The cortical processing of perceived emotion: A DC-related study on affective speech prosody. NeuroReport, 8, 623-627.

Imaizumi, S., Mori, K., Kiritan, S., Kawashma, R., Sugiura, M., FUKUDA, H., ET AL. (1997). Vocal identification of speaker and emotion activates different brain regions. NeuroReport, 8, 2809-2812.

JÄNCKE, L., VogT, J., Musial, F., LuTZ, K., \& KalveraM, K. T. (1996). Facial EMG responses to auditory stimuli. International Journal of Psychophysiology, 22, 85-96.

JUSLIN, P. N., \& LAUKKA, P. (2001). Impact of intended emotional intensity on cue utilization and decoding accuracy in vocal expression of emotion. Emotion, 1, 381-412.

Kemp, A. H., Silberstein, R. B., Armstrong, S. M., \& Nathan, P. J. (2004). Gender differences in the cortical electrophysiology processing of visual emotional stimuli. Neurolmage, 21, 632-646.

Kujala, T., Lepistö, T., Nieminen-von WENDT, T., NÄÄTÄNEN, P., \& NÄÄTÄNEN, R. (2005). Neurophysiological evidence for cortical discrimination impairment of prosody in Aspenger syndrome. Neuroscience Letters, 383, 260-265.

LANG, P. J., BRADLEY, M. M., \& CUTHBERT, B. N. (2005). International affective picture system (IAPS): Affective ratings of pictures and instruction manual. (Tech. Rep. A-6). Gainesville, FL: University of Florida.

Mehrabian, A., \& Russell, J. A. (1974). An approach to environmental psychology. Cambridge, MA: MIT Press.

Mikels, J. A., Fredrickson, B. L., LARKin, G. R., Lindberg, C. M., MAGLio, S. J., \& ReUTER-LoRENZ, P. A. (2005). Emotional category data on images from the International Affective Picture System. Behavior Research Methods, 37, 626-630. 
Pelli, D. G. (1997). The Video Toolbox software for visual psychophysics: Transforming numbers into movies. Spatial Vision, 10, 437-442.

Royet, J.-P., Zald, D., Versace, R., Costes, N., Lavenne, F., KoENIG, O., \& GeRVAIS, R. (2000). Emotional responses to pleasant and unpleasant olfactory, visual, and auditory stimuli: A positron emission tomography study. Journal of Neuroscience, 20, 7752-7759.

SANDER, K., BRECHMANN, A., \& SCHEICH, H. (2003). Audition of laughing and crying leads to right amygdala activation in a low-noise fMRI setting. Brain Research Protocols, 11, 81-91.

SANDER, K., \& SCHEICH, H. (2001). Auditory perception of laughing and crying activates human amygdala regardless of attentional state. Cognitive Brain Research, 12, 181-198.

SChIRMER, A., KoTz, S. A., \& FrIEDERICI, A. D. (2005). On the role of attention for the processing of emotions in speech: Sex differences revisited. Cognitive Brain Research, 24, 442-452.

Scott, K. S., Young, A. W., Calder, A. J., Hellawell, D. J., AggleTON, J. P., \& JoHNSON, M. (1997). Impaired auditory recognition of fear and anger following bilateral amygdala lesions. Nature, 385, 254-257.

Smith, C. A., \& Ellsworth, P. C. (1985). Patterns of cognitive appraisal in emotion. Journal of Personality \& Social Psychology, 48, 813-838.

STEVENSON, R. A., \& JAMES, T. W. (2007). [Subjective ratings of Ekman and Friesen's Pictures of Facial Affect according to dimensional and discrete emotional categories]. Unpublished raw data.

Stevenson, R. A., Mikels, J. A., \& James, T. W. (2007). Characterization of affective norms for English words by discrete emotional categories. Behavior Research Methods, 39, 1020-1024.

van Riun, S., Aleman, A., van Diessen, E., Berckmoes, C., VingerHOETS, G., \& KAHN, R. S. (2005). What is said or how it is said makes a difference: Role of the right fronto-parietal operculum in emotional prosody as revealed by repetitive TMS. European Journal of Neuroscience, 21, 3195-3200.

Verona, E., Patrick, C. J., Curtin, J. J., Bradley, M. M., \& Lang, P. J. (2004). Psychopathy and physiological reaction to emotionally evocative sounds. Journal of Abnormal Psychology, 113, 99-108.
Wrase, J., Klein, S., Gruesser, S. M., Hermann, D., Flor, H., ManN, K. ET AL. (2003). Gender differences in the processing of standardized emotional visual stimuli in humans: A functional magnetic resonance imaging study. Neuroscience Letters, 348, 41-45.

YiK, M. S. M., RUSSELL, J. A., \& BARRETT, L. F. (1999). Structure of self-reported current affect: Integration and beyond. Journal of Personality \& Social Psychology, 77, 600-619.

\section{ARCHIVED MATERIALS}

The following materials associated with this article may be accessed through the Psychonomic Society's Norms, Stimuli, and Data archive, www.psychonomic.org/archive.

To access these files, search the archive for this article using the journal name (Behavior Research Methods), the first author's name (Stevenson), and the publication year (2008).

FILE: Stevenson-BRM-(2008).zip.

DESCRIPTION: The compressed archive file contains five files:

Stevenson(2008)User_Manual.pdf, containing a .pdf file of the user manual.

Stevenson(2008)_allSubjects.txt, containing all subject information in plain text format.

Stevenson(2008)_female.txt, containing female information in plain text format.

Stevenson(2008)_male.txt, containing male information in plain text format.

Stevenson(2008).xls, containing the above information in Excel spreadsheet format.

AUTHOR'S E-MAIL ADDRESS: stevenra@indiana.edu

(Manuscript received February 26, 2007; revision accepted for publication May 25, 2007.) 